again accumulating in the abdomen. In this way she went on with, but little change, till the morning of April $23 \mathrm{rd}$. On that morning the flow of bile ceased suddenly, so that the dressing removed on the morning of the 24 th only contained a trace. The ascites had become so distressing that I tapped, drawing off on the afternoon of that day 294 ounces of a bile-stained fluid containing a considerable amount of blood. After this the record is that of a patient dying slowly, but without pain, of an incurable disease. On May 8th she died. The post-mortem examination revealed cancer of the pancreas and liver, especially the left lobe. The common bile duct was completely occluded, and perforation had occurred at its origin.

Remarks.-On reviewing the case, it appears to me that the tale of her illness as told by the patient is a history of biliary calculus. The intermittent character of the tumour she felt in her abdomen seemed rather to indicate calculus causing obstruction than stenosis of the duct from malignant disease; while the transitory jaundice referred to by her friend and the colicky pain shooting through to the back were alike characteristic of gall-stones. I expected to find a calculus causing obstruction of the common duct, and on sinding that the only stone present was fixed in the cystic duct, I concluded that its presence had occasioned hepatitis and catarrh of the common duct. The smooth regular border which the right lobe of the liver presented favoured this view. No doubt the ascites, the frequent pulse, and evening rise of temperature indicated a malignant origin On April 7th I telegraphed to Mr. Lawson Tait, desiring his opinion with regard to treatment of the fistulous opening in yiew of the excessive flow of bile. He very kindly replied at once, and wrote to me saying that the condition was new to him, but that he suspected malignant disease. The sudden stoppage of the flow of bile from the fistula was no doubt due to perforation of the common duct, and to the same cause we may attribute the presence of blood in the abdomen. The occurrence of monthly bleeding from the gums, which was noted by the patient, cannot, I think, be regarded as in any way related to menstruation, when we consider the pathological conditions presented by the case. Huelva, Spain.

\section{POISONING BY COCAINE ADMINISTERED SUBCUTANEOUSLY.}

\section{BY BERNARD PITTS, M.C.}

WITH reference to the annotation in THE LANCET of Dec. 3rd, on Poisoning by Cocaine, the following case may prove of interest. I am indebted for the careful notes to Mr. Calvert, assistant house-surgeon to St. Thomas's Hospital. My attention was called to the case in the out-patient room by Mr. Calvert as one of cocaine poisoning. At first sight the patient's condition suggested the probability of a nervous attack, but it was soon obvious that this was not a sufficient explanation. The symptoms shortly afterwards became alarming, and gave rise to grave apprehension as to the result. There is no reason to suppose that the injection in this case was made into a vein, and the preparation of cocaine was the same as had been used in other cases in the out-patient room. The drug is now in very general use for minor operations, and few cases have been recorded where toxic effects have ensued. I have several times noticed glight indications of constitutional effect, but used the drug oftentimes much more freely than in this case, without anxiety. I can now no longer regard the drug as free from risk, and shall be more cautious for the future, especially when the patient has not had the drug tried on him before, for there must doubtless be some peculiar idiosyncrasy in the subject when such marked symptoms arise.

The patient, a boy aged thirteen, came to the out-patient room at St. Thomas's Hospital on Saturday, Oct. 22nd, suffering from a suppurating sebaceous cyst situated in the middle of the forehead. This had previously been opened by a local practitioner some time prior to his application at the hospital, but the incision having healed the matter had reaccumulated. It was therefore decided to evacuate the contents and scrape out the interior of the cavity under cocaine. For this purpose two grains of hydrochlorate of cocaine were dissolved in thirty-five minims of water, and of this solution twenty-five minims were injected through two punctures, one on each side of and close to the abscess cavity. The solution was injected at 2.30 P.M. Within five minutes of the injection, the patient became pale and very restless, with hurried respiration and a rapid pulse (120). The abscess was quickly incised, the contents evacuated, the interior scraped, and the wound dressed. The symptoms shortly afterwards became alarming. The patient was exceedingly restless, constantly shifting his position, throwing his arms and legs about, and wringing his hands. The heart's action became tumultuous; the pulse at the wrist was irregular, rapid, weak, and almost imperceptible; the respirations were rapid, shallow, and irregular; and the pupils were dilated. The patient appeared in great distress; removed his collar and unbuttoned his shirt, so as to expose the chest. Meanwhile, balf a drachm of aromatic spirit of ammonia was administered, and, the symptoms continuing, the dose was repeated a fow minutes afterwards. Nausea and retching, with eructations, set in at 3.15 P.M., the patient complaining of great pain in his "stomach." This pain was markedly increased on firm pressure over the epigastrium, but was relieved by friction. Half an ounce of brandy was administered, and friction applied to the epigastrium. The patient's condition improved; the pulse, though rapid, became stronger, and the respiration slower and more regular. The dose of brandy was repeated, and a mustard poultice was applied to the epigastrium for fifteen minutes. The symptoms at 4 P.M. continued as before, but were less marked. The patient still complained of pain in his stomach, and lay on his left side with his legs drawn up. He said " he felt better," and was not so restless. At 4.45 P.M. he was removed to the ward, placed in a warmed bed, and hot bottles applied to hisfeet. He now improved rapidly. He was sick and vomited before 6 P.M., after which there was little to record.

The patient never lost consciousness, and was quite rational when spoken to throughout the persistence of the symptoms. The local anæsthesia was certainly not well marked at the time of the operation; afterwards it was not tested. Taking twenty-five minims of the solution as injected, this would give one grain and a half of cocaine, but owing to some of the solution being spilled and some escaping from the punctures on withdrawal of the syringe, it is in excess of the amount actually injected.

Harley-street, W.

\section{Clinical a dotes:}

MEDICAL, SURGICAL, OBSTETRICAL, AND THERAPEUTICAL.

CONGENITAL MALFORMATION OF FOREARMS AND HANDS, WITH CONCOMITANT ATROPHY OF THE BICEPS AND BRACHIALIS ANTICUS MUSCLES.

BY G. C. W ILKIN, M.R.C.S., L.S.A.

THE following case came under my observation whilst off the coast of China on board the steamship Palamed:-

The man, a Chinese coolie, was about five feet in height, and in appearance remarkable for his childish expression and the absence of a pig-tail, such hair as was present on his scalp being short, grey, and stubbly. His lower limbs and body were perfectly normal. His hands, both of which were small, consisted of the four fingers and their corresponding bones and muscles, the thumbs being absent. There was a difference in the arrest of development on the two sides. Right side: Carpus-slight trace of trapezium and scaphoid bones; entire absence of pisiform bone. Forearm-Styloid process of the radius absent. Styloid process of the ulna hypertrophied. Both bones dislocated backwards and outwards from the humerus. Humerus normal. Biceps and brachialis anticus small, but fairly well nourished. Left side: Carpus-Absence of trapezium and scaphoid, also of the pisiform bone. Forearm-Radius an inch and a half long, joining the ulna at its lower extremity, its upper extremity being dislocated outwards. Ulna from the junction of the radius to the styloid process hypertrophied; above this normal. Humerus convexly curved forwards and outwards. Entire absence of the biceps and brachialis anticus muscles. There was very slight power over the movenents at the wrists, but the fingers $w \in$ re well controlled by their muscles.

The family' history was difficult to obtain. He had no 